\title{
DONNELLAN'S DISTINCTION AND A COMPUTATIONAL MODEL OF REFERENCE
}

\author{
Artificial Intelligence Center \\ Amichai Kronfeld \\ SRI International \\ 333 Ravenswood Avenue \\ and \\ Center for the Study of \\ Menlo Park, CA 94025 \\ kronfeld@sri-warbucks \\ Language and Information \\ Stanford University \\ Stanford, CA 94305
}

\begin{abstract}
In this paper, I describe how Donnellan's distinction between referential and attributive uses of definite descriptions should be represented in a computational model of reference. After briefly discussing the significance of Donnellan's distinction, I reinterpret it as being three-tiered, relating to object representation, referring intentions, and choice of referring expression. I then present a cognitive model of referring, the components of which correspond to this analysis, and discuss the interaction that takes place among those components. Finally, the implementation of this model, now in progress, is described.
\end{abstract}

\section{INTRODUCTION}

It is widely acknowledged that Donnellan's distinction [7] between referential and attributive uses of definite descriptions must be taken into account in any theory of reference. There is not yet agreement, however, as to where the distinction fits in a theoretical model of definite noun phrases. For Cohen [4], the intention that the hearer identify a referent constitutes a crucial difference between the referential and the attributive. Barwise and Perry [3], on the other hand, treat their value-loaded/value-free distinction as the central feature of the referential versus the attributive. However, as pointed out by Grosz et al. [9], this analysis ignores an essential aspect of Donnellan's distinction, namely, the speaker's ability, when using a description referentially, to refer to an object that is independent of the semantic denotation.

The problem of determining the correct interpretation of Donnellan's distinction is of considerable importance. First, Donnellan's distinction seems to violate the principle that reference to physical objects is achieved by virtue of the descriptive content of referring expressions. This principle can be found practically everywhere - for example, in Frege's sense and reference, Rusell's theory of descriptions, and Searle's speech acts. In the referential use of definite descriptions, however, reference seems to be established independently of descriptive content. If I say "The man over there with a glass of white wine is...," I may be successful in my act of referring - regardless of whether the person over there is a man or a woman, the glass is full of wine or grape juice, the color of the beverage is white or red, and so on. This, if accepted, has far-reaching consequences for the meaning of referring ex- pressions, for the logical structure of propositions, and for the theory of propositional attitudes.

Second, the referential/attributive distinction forces us to reconsider the division between semantics and pragmatics. It seems that a speaker's intentions in using a referring expression do make a semantic difference. If I say "Smith's murderer is insane," meaning that whoever murdered Smith is insane (the attributive case), what I say is true if and only if the one and only murderer is insane. If, on the other hand, my intention is to use the definite description referentially (referring to, say, Tom, who is accused of being the culprit), what I say is true if and only if Tom is indeed insane - whether he is the murderer or not. Unless we understand the interaction between conventional meaning and a speaker's intentions in such cases, we cannot hope to construct an adequate model of referring and language use in general.

Finally, Donnellan's distinction brings to the fore the role of identification in the speech act of referring. Both Strawson and Searle $([17,16])$ attempted to analyze referring in terms of identification and identifying descriptions. But Donnellan has pointed to what seems to be a clear distinction between cases in which identification is required (referential use) and those in which it is not (attributive use). This calls for a new analysis of the speech act of referring, one that does not rely on identification as a central concept. ${ }^{I}$

ln this paper, I present a general framework for treating Donnellan's distinetion. In particular, I contend the following:

1. The apparent simplicity of the referential/attributive distinction masks three aspects of the problem of reference. In a sense, it is not one distinction but three: the first has to do with representations of objects, the second with referring intentions, the third - with the choice of referring expressions.

2. These three distinctions are independent of one another, and should be handled separately. Each is relevant to a different component of a plan-based model of reference: the dats base, the planner, and the utterance generator, respectively.

3. Although the three distinctions are mutually independent,

\footnotetext{
'These comments, naturally, only touch the surface. For an extensive discussion of the significance of the referential/attributive distinction see my thesis [14]. For a discussion of the role of identification in referring. see the paper coauthored by Appelt and me on this topic [2].
} 
they of course interact with one another. The notion of a conversationally relevant description provides a basis for explaining how the interaction operates.

In the following sections, the three aspects are presented, their interactions discussed, and an initial attempt to achieve an implementation that takes them into account is described.

\section{CRITERIA}

How is the referential to be distinguished from the attributive? Two criteria are usually offered:

1. Even though, when used attributively, the description must denote the intended referent, in the referential use this is not necessary.

2. In the referential use, the speaker has a particular object in mind, whereas in the attributive he does not.

These criteria have been taken to be equivalent: any use of a definite description that is referential according to one criterion should also be classified as referential according to the other (and similarly for the attributive use). However, the equivalence of the two criteria is really an illusion: some uses of definite descriptions are referential according to one criterion, but attributive according to the other. For example, let us suppose that John, a police investigator, finds Smith's murdered body, and that there are clear fingerprints on the murder weapon. Now consider John's utterance: "The man whose fingerprints these are, whoever he is, is insane." Note that John intended to speak of Smith's murderer, and he may very well have been successful in conveying his intended referent, whether or not the fingerprints indeed belonged to the murderer. Hence, according to the first criterion, the description, "The man whose fingerprints these are," was used referentially. On the other hand, John did not have any particular person in mind. Hence, according to the second criterion, the description must have been used attributively.

Many, including Donnellan, regard the second criterion as the more significant one. But even this criterion is given two conflicting interpretations. On the one hand, "having a particular object in mind" is taken as an epistemic concept: this view holds that one can have a particular object in mind while referring only if one knows who or what the referent is. On the other hand, the criterion also receives what I call the modal interpretation. According to this reading, the referential use of a definite description is simply tantamount to employing the description as a rigid designator. Obviously, the two interpre. tations are not equivalent. As Kaplan demonstrates [11], one can use a description as a rigid designator without having any idea who the referent is.

Thus, there are three aspects of Donnellan's distinction that should be carefully separated. These aspects can be represented in terms of three dichotomies:

- Having knowledge of an object versus not having such knowledge (the epistemic distinction).

- Using a description as a rigid designator versus using it as a nonrigid one (the modal distinction).
- Using a definite description "the $\phi^{\text {" }}$ to refer to whoever or whatever the $\phi$ may be, versus using "the $\phi$ " to refer to an object $x$, whether or not $x$ is indeed the $\phi$ (the speech act distinction).

\section{THREE COMPONENTS}

The epistemic, modal, and speech act distinctions correspond to three components that a plan-based model of reference must possess. ${ }^{2}$ Any such model must contain the following:

1. A database that includes representations of objects

2. A planner that constructs strategies for carrying out referring intentions

3. An utterance generator that produces referring expressions

Let us call these the database, the planner, and the utterancegenerator, respectively. The next three sections describe a cognitive model of referring that incorporates these components.

\section{Object Representations}

Objects are represented to agents by terms. These terms are grouped into individuating sets. An individuating set for an agent $S$ is a maximal set of terms, all believed by $S$ to be denoting the same object. For example, for John, the police investigator, the set $\{$ Smith's murderer, the man whose fingerprints these are $\}$ is an individuating set of Smith's murderer. The incredibly complex cluster of internal representations under which, for instance, John's mother would be represented to him is also an individuating set, although it would be impractical to enumerate all the terms in this set.

An individuating set is grounded if it contains either a perceptual term or a term that is the value of a function whose argument is a perceptual term. For example, a set containing the description "your father" is grounded, since it contains a terms that is the result of applying the function FATHER-OF to a perceptual term representing you.

It should be emphasized that an individuating set is the result of the speaker's beliefs, not a mirror of what is actually the case. A speaker may possess two distinct individuating sets that, unbeknownst to him, determine the same object (e.g., Oedipus's representations of his mother and his wife). On the other hand, a speaker may possess an individuating set containing two or more terms that actually denote different objects. Moreover, the object that an agent believes to be denoted by the terms of some individuating set may not exist in the actual world.

Whether or not an agent can have knowledge of the referent, or know who or what the referent is (the epistemic distinction), depends on the nature of the relevant individuating set. In a computational model, we can place a number of restrictions on individuating sets to reflect various epistemological intuitions. For example, we may require that, for an agent to be able to manipulate an object, the relevant individuating set must contain a perceptual term, or that, for an agent to know

\footnotetext{
${ }^{2}$ For a plan-based model of referring, definite noun phrases, and speech acts in general, see articles by $A$ ppelt, Cohen, Cohen and Levesque, Cohen and Perrault $([1,4,5,6])$.
} 


\begin{tabular}{|c|c|c|}
\hline DISTINCTION & INTERPRETATION & COMPONENT \\
\hline \hline Epistemic & Type of individuating set & Database \\
\hline Modal & Type of referring intentions & Planner \\
\hline Speech act & Choice of definite noun phrase & Utterance generator \\
\hline
\end{tabular}

Table 1: Donnellan's distinction, its interpretation(s), and the corresponding computational components.

who a certain person is (relative to purpose $P$ ), the relevant individuating set must include a privileged term determined by $P$, or that, for an agent to have knowledge of an object, the relevant individuating set must be grounded, and so on.

Since individuating sets are part of the database, this is where the epistemic distinction belongs.

\section{Referring Intentions}

A speaker may have two distinct types of referring intentions. First, he may select a particular term from the relevant individuating set, and intend this term to be recognized by the hearer. Second, the speaker may intend to refer to the object determined by an individuating set, without intending any particular term from the set to be part of the proposition he wants to express. Consider, for example, the following two statements:

\section{The author of Othello wrote the best play about jealousy.}

2 Shakespeare was born in Stratford-upon-Avon.

In making both statements, a speaker would normally be referring to Shakespeare. But note the difference in referring intentions between the two: in the first statement, the speaker selects a particular aspect of Shakespeare, namely, the fact that he is the author of Othello, and intends the hearer to think of Shakespeare in terms of this aspect. In the second statement, the speaker does not select any particular aspect of Shakespeare from the relevant individuating set. Indeed, he may not care at all how the hearer makes the connection between the name "Shakespeare" and the referent.

The two types of referring intentions yield two distinct types of propositions. When the speaker does not intend any par. ticular aspect of the referent to be recognized by the hearer, the proposition expressed in this way is singular, that is, it does not contain any individual concept of the referent. Consequently, the referring expression chosen by the speaker (be it a proper name, a demonstrative, or even a definite description) is used as a rigid designator, which means that it picks out the same individual in all possible worlds where the referent exists. On the other hand, if a particular aspect of the referent is meant to be recognized by the hearer, then the individual concept corresponding to that aspect is part of the proposition expressed and should therefore be taken into account in evaluating the truth value of what is said. Thus, it is the speaker's referring intentions that determine whether or not he will use a definite description as a rigid designator (the modal distinction). Since referring intentions are represented in the planner, this is where the modal distinction belongs.

Note that the two types of referring intentions can be described as intentions to place constraints on the way the hearer will be thinking of the referent. In Appelt and Kronfeld [2], this is generalized to other referring intentions - for example, the intention that the hearer identify the referent.

\section{Referring Expressions}

Once the speaker decides what his referring intentions are, he must choose an appropriate referring expression. Usually, if a particular aspect of the referent is important, a suitable definite description is employed; otherwise a proper name or a demonstrative may be more useful. However, such a neat correlation between types of referring expressions and referring intentions may not happen in practice. In any case, as we shall see in the next section, the speaker's choice of a referring expression constitutes an implicit decision as to whether the denotation of the referring expression must coincide with the intended referent (the speech act distinction). The choice of referring expression is naturally made within the utterance generator, where the speech act distinction is represented.

By way of summary, Table 1 shows how Donnellan's distinction, in its reinterpreted form, is related to a plan-based model of reference.

\section{RELEVANT DESCRIPTIONS}

Kripke and Searle $[12,15]$ explain the referential use as a case in which speaker's reference is distinct from semantic reference. This leaves an important question unanswered: why must speaker's reference and semantic reference coincide in the attributive use? ${ }^{3}$

Sometimes two definite descriptions are equally useful for identifying the intended referent, yet cannot be substituted for each other in a speech act. The description employed, besides being useful for identification, has to be relevant in some other respect. Consider the utterance: "New York needs more policemen." Instead of "New York," one might have used "The largest city in the U.S." or "The Big Apple," but "The city hosting the $1986 \mathrm{ACL}$ conference needs more policemen" won't do, even though this description might be as useful in identifying New York as the others. The latter statement simply conveys an unwarranted implication.

As a generalization, we may say that there are two senses in which a definite description might be regarded as relevant. First, it has to be relevant for the purpose of letting the hearer know what the speaker is talking about. ${ }^{4}$ A description that is relevant in this sense may be called functionally relevant. Second, as the example above indicates, a description might exhibit a type of relevance that is not merely a referring tool.

\footnotetext{
${ }^{3}$ As redefined by the spech act distinction.

${ }^{4}$ Whether the hearer is also expected to identify the referent is a sepa. rate issue.
} 
A description that is relevant in this noninstrumental sense might be called conversationally relevant.

Every use of a definite description for the purpose of reference has to be functionally relevant. But not every such use has to be conversationally relevant. If indicating the referent is the only intended purpose, any other functionally relevant description will do just as well.

In other cases, the description is supposed to do more than just point out the intended referent to the hearer. Consider the following examples:

\section{This happy man must have been drinking champagne.}

4 The man who murdered Smith so brutally has to be insane.

\section{The winner of this race will get $\$ 10,000$.}

In these examples, the speaker implicates (in Grice's sense) something that is not part of what he says. In (3), it is implicated that the man's happiness is due to his drinking. In (4), it is implicated that the main motivation for believing the murderer to be insane is that he committed such a brutal homicide. The implicature in (5) is that the only reason for giving the winner $\$ 10,000$ is his victory in a particular race. In all these cases, what is implicated is some relationship between a specific characteristic of the referent mentioned in the description and whatever is said about that referent. In such cases, it does matter what description is chosen, since the relevance is both functional and conversational. No other description, even if it identifies equally well, can be as successful in conveying the intended implicature.

The conversationally relevant description may not be mentioned explicitly, but rather inferred indirectly from the context. In the fingerprint example, the speaker uses the description, The man whose fingerprints these are, but the conversationally relevant description is nevertheless Smith's murderer.

Thus, there are three general ways in which a speaker may employ a referring definite description:

1. If the discourse requires no conversationally relevant description, any functionally relevant one will do. This covers all standard examples of the referential use, in which the sole function of the definite description is to indicate an object to the hearer.

2. If a conversationally relevant description is needed, the speaker may do either of the following:

(a) Use the description explicitly. This is what is done in standard examples of the attributive use.

(b) Use a different, functionally relevant description. The speaker can do so, however, only if the context indicates the aspect of the referent that corresponds to the conversationally relevant description. This explains the ambiguity of the fingerprint example. As the definite description uttered is only functionally relevant, its use appears to be referential. Yet, unlike the referential case, a conversationally relevant description is implied.
In sum, when the description used is conversationally relevant, the speaker intends that the specific way he chose to do his referring should be taken into account in interpreting the speech act as a whole. Consequently, if the description fails, so does the entire speech act. On the other hand, if the description is only functionally relevant, the context may still supply enough information to identify the intended referent.

\section{INTERACTIONS}

When a speaker plans a speech act involving reference to an object, he must determine whether or not a conversationally relevant description is needed. However, the nature of the individuating set, on the one hand, and constraints on choices of referring expressions, on the other, may influence the speaker's planning in various ways. For example, if the individuating set contains only one item, say, the shortest spy, the definite description "the shortest spy" must be conversationally relevant. This is true both on formal and pragmatic grounds. From a formal standpoint, the description is conversationally relevant by default: no other functionally relevant description can be substituted because no such description is available. From a pragmatic standpoint, the description "the shortest spy" is very likely to be conversationally relevant in real discourse, simply because all we know about the referent is that he is the shortest spy. Thus, whatever we may have to say about that person is very likely to be related to the few facts contained in the description.

Even if it is clear that a conversationally relevant description is needed for the speech act to succeed, constraints on choices of referring expressions may prevent the speaker from using this description. One such constraint results from the need to identify the referent for the hearer. If the conversationally relevant description is not suited for identification, a conflict arises. For example, in "John believes Smith's murderer to be insane," the speaker may be trying simultaneously to represent the content of John's belief and to identify for the hearer whom the belief is about. Sometimes it is impossible to accomplish both goals with one and the same description.

\section{IMPLEMENTATION}

This paper is part of an extensive analysis of the referential/attributive distinction, which I use in the construction of a general model of reference [13]. My ultimate research objective is to provide a computational version of the reference model, then to incorporate it into a general plan-based account of definite and indefinite noun phrases. An experimental program that implements individuating sets has already been written. Called BERTRAND, this program interprets a small subset of English statements, and stores the information in its database, which it then uses to answer questions. Individuating sets are represented by an equivalence relation that holds among referring expressions: two referring expressions, $R_{1}$ and $R_{2}$, belong to the same individuating set if, according to the information interpreted so far, $R_{1}$ and $R_{2}$ denote the same object. In construeting individuating sets, BERTRAND uses a combination of logical and pragmatic strategies. The logical strategy exploits the fact that the relation "denote the same object" is symmetric, transitive, and closed under substitution. Thus, it 
can be concluded that two referring expressions, $R_{1}$ and $R_{2}$, denote the same object (belong to the same individuating set) in one of the following ways: ${ }^{5}$

1. Directly, when the statement " $R_{1}$ is $R_{2}$ " (or " $R_{2}$ is $R_{1}{ }^{\text {”) }}$ has been asserted.

2. Recursively using transitivity - i.e., when, for a referring expression $R_{5}$, it can be shown that $R_{1}$ and $R_{5}$, as well as $R_{3}$ and $R_{2}$, belong to the same individuating set.

3. Recursively using substitution - i.e., when $R_{1}$ and $R_{2}$ are identical, except that $R_{1}$ contains a referring expression $s u b R_{1}$ exactly where $R_{2}$ contains a referring expression $s u b R_{2}$, and $s u b R_{1}$ and $s u b R_{2}$ belong to the same individuating set.

Note that, in the logical strategy, it is tacitly assumed that the relation of denoting the same object always holds between two identical tokens of referring expressions. This is obviously too strong an assumption for any realistic discourse: for example, two utterances of "The man" may very well denote two different people. On the other hand, the logical strategy fails to capture cases in which it is implied (although never actually asserted) that two distinct referring expressions denote the same thing. For example, "I met Marvin Maxwell yesterday. The man is utterly insane!"

To compensate for these weaknesses, BERTRAND uses a strategy based on Grosz's notion of "focus stack" $[8,10]$. In conceptual terms (and without going into details), it works as follows: a stack of individuating sets, representing objects that are "in focus," is maintained throughout the "conversation." When a new referring expression is interpreted, it is transformed into an open sentence $D(x)$ with a single free variable $x .^{6}$ An individuating set $I$ is said to subsume an open sentence $S$ if $S$ can be derived from $I$. The first individuating set in the focus stack to subsume $D(x)$ represents the object denoted by the new referring expression. This solves the aforementioned problems: two occurrences of the same referring expression are considered as denoting the same object only if both are subsumed by the same individuating set in the focus stack, and two distinct referring expressions may still be considered as denoting the same object even though the logical strategy failed to show this, provided that both are subsumed by the same individuating set.

Once the concept of an individuating set has been implemented, referring intentions can be represented as intentions to activate appropriate subsets of individuating sets. For example, the intention to use a conversationally relevant description can be represented as the plan to activate a subset of an individuating set that contains the term associated with the description. This is the topic of a current joint research effort with D. Appelt [2] to investigate the interaction that takes place between individuating sets and Appelt's four types of

\footnotetext{
${ }^{5}$ What belongs to an individuating set, of course, is not a referring expression but the logical structure associated with it. For the sake of simplicity, however, I do not make this distinction here.

"For example, "The man from the city by the bay" is transformed into
}

\section{$\operatorname{Man}(x) \& \operatorname{From}\left(x, X_{i}\right)$}

where $X_{i}$ is an "internal symbol" associated with $\operatorname{City}(y) \& \mathbf{B y}\left(y, X_{j}\right)$, and $X$, is associated with $\operatorname{Bay}(z)$. concept activation actions [1]. The next stage in the development of BERTRAND - the implementation of referring intentions - will be based on this research. In the final stage, individuating sets and referring intentions will be used to generate actual referring expressions.

\section{ACKNOWLEDGMENTS}

This research was supported by the National Science Foundation under Grant DCR-8407238. I am very grateful to Doug Appelt and Barbara Grosz for detailed comments on earlier drafts, as well as to members of the Discourse, Intention and Action seminar at the Center for the Study of Language and Information for stimulating discussions of related issues.

\section{REFERENCES}

[1] Douglas E. Appelt. Some pragmatic issues in the planning of definite and indefinite noun phrases. In Proceedings of the 23rd Annual Meeting, Association for Computational Linguistics, 1985.

[2] Douglas E. Appelt and Amichai Kronfeld. Toward a model of referring and referent identification. Forthcoming. Submitted to the AAAI convention, Philadelphia, August 1986.

[3] Jon Barwise and John Perry. Situations and Attitudes. The Massachsetts Institute of Technology Press, Cambridge, Massachusetts, 1983.

[4] Philip R. Cohen. Referring as requesting. In Proceedings of the Tenth International Conference on Computational Linguistics, pages 207-211, 1984.

[5] Philip R. Cohen and Hector Levesque. Speech acts and the recognition of shared plans. In Proceedings of the Third Biennial Conference, Canadian Society for Computational Studies of Intelligence, 1980.

[6] Philip R. Cohen and C. Raymond Perrault. Elements of a plan-based theory of speech acts. Cognitive Science, 3:117-212, 1979.

[7] Kieth S. Donnellan. Reference and definite description. Philiosophical Review, 75:281-304, 1966.

[8] Barbara J. Grosz. Focusing and description in natural language dialogues. In A. Joshi, I. Sag, and B. Webber, editors, Elements of Discourse Understanding, pages 85105, Cambridge University Press, Cambridge, England, 1980.

[9] Barbara J. Grosz, A. Joshi, and S. Weinstein. Providing a unified account of definite noun phrases in discourse. In Proceedings of the Twenty-first Annual Meeting, pages 44-50, Association for Computational Linguistics, 1983.

[10] Barbara J. Grosz and Candace L. Sidner. Discourse structure and the proper treatment of interruptions. In Proceedings of the Ninth International Joint Conference on Artificial Intellignece, pages 832-839, 1985. 
[11] David Kaplan. Dthat. In Peter Cole, editor, Syntax and Semantics, Volume 9, Academic Press, New York, New York, 1978.

[12] Saul Kripke. Speaker reference and semantic reference. In French et al., editor, Contemporary Perspectives in the Philosophy of Language, University of Minnesota Press, Minneapolis, Minnesota, 1977.

[13] Amichai Kronfeld. Reference and Denotation: The Descriptive Model. Technical Note 368, SRI International Artificial Intelligence Center, 1985.

[14] Amichai Kronfeld. The Referential Attributive Distinetion and the Conceptual-Descriptive Theory of Reference. PhD thesis, University of California, Berkeley, 1981.

[15] John Searle. Referential and attributive. In Expression and Meaning: Studies in the Theory of Speech Acts, Cambridge University Press, Cambridge, England, 1979.

[16] John Searle. Speech Acts: An Essay in the Philosophy of Language. Cambridge University Press, Cambridge, England, 1969.

[17] Peter F. Strawson. On referring. In J.F Rosenberg and C. Travis, editors, Reading in the Philosophy of Language, Prentice Hall, Englewood, New Jersey, 1971. 